\title{
LetTers
Is stavudine worth saving?
}

To the Editor: The ultimate goal of HIV therapy in resource-constrained settings must be to keep as many people alive with the best possible quality of life using the resources available. The question debated between Andrieux-Meyer et al. ${ }^{1}$ and Venter et al. ${ }^{2,3}$ might therefore be: 'With the resources available, can we keep more people alive with the best possible quality of life using stavudine $20 \mathrm{mg}$ bd or tenofovir $300 \mathrm{mg}$ od as standard first line therapy?' Quality of life is extremely important but unlikely to be the overriding factor if budgetary constraints restrict access to ART and therefore increase mortality. Both groups seem to agree that tenofovir is superior to stavudine for most patients and that the crux of the argument is about cost. Venter et al. describe tenofovir as 'the gold standard' and state that 'these arguments [about the benefits of tenofovir] are likely to be irrelevant when the cost of medication is considered? Andrieux-Meyer et al. acknowledge that 'the rationale for this [proposed] trial is to lower treatment costs'

The question of whether stavudine or tenofovir will ultimately save the most lives within the budget is complex. Many of the variables change over time and differ between countries. The 'resources available' may even be influenced by the choice of ART regimens if, for example, a government tries to save money by using inferior but cheaper drugs. In some settings, the rate-limiting factors for saving lives may not actually be financial resources to buy medications but a lack of human resources or logistic challenges. Despite these complexities, it is important that each side provides as clear a picture as possible. In addition to cost-effectiveness estimates, we need absolute cost estimates for competing regimens including the estimated minimum cost of tenofovir once costs are driven primarily by raw materials. We need estimates of the number of patients requiring ART over time, and we need to know how much funding is available. In particular, we need to know how the recent cuts to funding from donors such as the Global Fund will affect the provision of ART in different countries. Without such figures, any discussion about drugs in phase II trials that have a high attrition rate and long time delay before affordability is frankly irrelevant.

Venter et al. have conceded that 2 years may be too short to show differences between the groups, and it will be vital to motivate for lengthening the trial if non-inferiority is shown at 2 years. Upon completion of the trial, it would seem sensible to use the cheaper option if noninferiority is shown. However, even if stavudine is not non-inferior (i.e. is inferior), it still might be preferred in some settings if the alternative is running out of money and restricting access to ART. We commonly use inferior treatments owing to cost constraints; just one example is the use of amphoteracin B monotherapy to treat cryptococcal meningitis rather than the superior but more expensive combination of liposomal amphoteracin B and flucytosine. Activists will argue that we should continue to lobby for increases in funding and reductions in drug cost. Of course we should, but we must also be mindful to look at the problem through the eyes of future patients. They will not thank us if our lobbying efforts fail to prevent ART rationing owing to shortfalls in funding.

It is clear that both sides of the debate have the best interests of patients in mind. To make an informed decision on the merits of trialling stavudine against tenofovir in the Southern African context, we need a clearer description of the costs of each strategy and the likely available resources. In short, we need to know whether choosing tenofovir over stavudine in first-line therapy is likely to lead to restricted access to care in some settings.

\section{Tom H Boyles}

Division of Infectious Diseases \& HIV Medicine

Department of Medicine

Groote Schuur Hospital and University of Cape Town

\section{REFERENCES}

1. Andrieux-Meyer I, Clayden P, Collins S, et al. Why it's time to say goodbye to stavudine... everywhere. Southern African Journal of HIV Medicine 2012;13:17-19.

2. Innes $S$, Cotton M, Venter F. Why should we still care about the stavudine dose? Southern African Journal of HIV Medicine 2011;12:14-15.

3. Venter F, Innes S, Cotton M. Low-dose stavudine trials: a public health priority for developing countries. Southern African Journal of HIV Medicine 2012;13:20-21. 